\title{
Análise do Controle de Qualidade das Biópsias e Produtos de Cirurgia de Alta Frequência no Programa de Prevenção do Câncer do Colo do Útero do Estado do Paraná, Brasil Analyzing Quality Control of Biopsies and Loop Electrosurgical Excisional Procedure Specimens Performed at the Cervical Cancer Prevention Program in the State of Paraná, Brazil \\ Análisis de Control de Calidad de las Biopsias y Productos de Cirugía de Alta Frecuencia en el Programa de Prevención del Cáncer del Cuello del Útero en el Estado de Paraná, Brasil
}

\author{
Luiz Martins Collaço' ; Larissa Uhlmann Wendling ${ }^{2}$; Lucia de Noronha ${ }^{3}$; Joel Totsuguij ${ }^{3}$ Ana Paula Martins Sebastião ${ }^{4}$; Samuel Regis Araúi $0^{5}$; \\ Avelino Hass ${ }^{5}$
}

\begin{abstract}
Resumo
Introduçáo: O seguimento e o tratamento adequados das lesôes neoplásicas e pré-neoplásicas do colo do útero dependem do diagnóstico histopatológico correto. A instauração de mecanismos de controle de qualidade para esse tipo de procedimento diagnóstico é fundamental para garantir precisão aos exames. Objetivo: Comparar o diagnóstico inicial de biópsias e peças cirúrgicas removidas por cirurgia de alta frequência, fornecido por um laboratório da rede de serviço, com o diagnóstico revisado pela unidade de monitoramento de qualidade da Associação Paranaense de Patologia, identificando quais as discrepâncias mais prevalentes. Método: Estudo transversal observacional com revisão do banco de dados da unidade de Controle de Qualidade referente ao ano de 2010 para avaliar o percentual de concordância geral, para cada laboratório e as discrepâncias diagnósticas mais comuns em produtos de biópsias e cirurgias de alta frequência do colo uterino. A concordância foi avaliada através do índice Kappa. Resultados: A compatibilidade entre o diagnóstico inicial e do controle de qualidade foi de 98,6\%, variando por laboratório entre 92,9\% e 100\%. As discrepâncias diagnósticas encontradas foram entre lesão intraepitelial de baixo grau e cervicite $(36,3 \%)$ e lesão intraepitelial de baixo grau e lesão intraepitelial de alto grau (27,3\%). Para todas as categorias diagnósticas estudadas, foi encontrado índice Kappa de 0,99. Conclusão: As discrepâncias diagnósticas mais comuns foram entre lesão intraepitelial de baixo grau e diagnóstico de lesões benignas (supradiagnóstico) e entre lesão intraepitelial de baixo grau e lesão intraepitelial de alto grau (subdiagnóstico). As taxas de concordância encontradas são consideradas excelentes. Palavras-chave: Controle de Qualidade; Neoplasias do Colo do Útero/diagnóstico; Neoplasias do Colo do Útero/patologia; Lesôes Pré-Cancerosas/diagnóstico; Conização; Neoplasia Intra-Epitelial Cervical/patologia
\end{abstract}

\footnotetext{
${ }^{1}$ Médico Patologista. Membro da Unidade de Monitoramento Externo da Qualidade (UMEQ) - Associação Paranaense de Patologia (APP). Professor da cadeira de Patologia da Faculdade Evangélica do Paraná.

${ }^{2}$ Acadêmica do $10^{\circ}$ período do Curso de Medicina da Faculdade Evangélica do Paraná.

${ }^{3}$ Médico Patologista. Membro da UMECQ-APP. Professor da Cadeira de Patologia da Universidade Federal do Paraná.

${ }^{4}$ Médica Patologista. Membro da UMECQ-APP. Professora da Cadeira de Patologia da Universidade Positivo.

${ }^{5}$ Médico Patologista. Membro da UMECQ-APP.

Enderȩ̧o para correspondência: Luiz Martins Collaço. Rua Padre Agostinho, 1477 -Bigorrilho. Curitiba (PR), Brasil. CEP: 80710-0000. E-mail:1mcollaco@uol.com.br.
} 


\section{INTRODUÇÃO}

O câncer do colo do útero é uma das neoplasias malignas mais frequentes na população feminina. Estima-se que em 2012 haverá 17.540 novos casos em todo o Brasil e 770 casos no estado do Paraná, incidência menor somente do que a do câncer da mama, de cólon e reto e de pulmão ${ }^{1}$. A evolução do câncer do colo do útero geralmente é lenta e os aspectos epidemiológicos e etiológicos (que incluem a relação com a infecção por diferentes tipos de Papilomavirus humano, o HPV) estáo bem definidos, bem como a evolução do processo, que possui fases pré-invasivas, conhecidas como neoplasias intraepiteliais cervicais (NIC) e fases invasivas, malignas, onde estão as lesôes microinvasoras ou invasoras ${ }^{2}$.

O estabelecimento do diagnóstico precoce das neoplasias do colo do útero, por meio do exame citopatológico (teste de Papanicolaou), permite o rastreamento das lesôes nas fases iniciais ${ }^{2}$ e o diagnóstico nessas fases proporciona uma maior chance de cura para a portadora da lesão, além da utilização de métodos terapêuticos menos agressivos. Um dos fatores relacionados ao sucesso no rastreamento dessa doença é a acuidade diagnóstica do exame citopatológico ${ }^{3}$.

No Brasil, o Ministério da Saúde faz o controle do câncer do colo do útero por meio Programa Nacional de Controle do Câncer do colo do Útero e Mama (Viva Mulher). Curitiba foi uma das cinco cidades brasileiras pioneiras na implantação desse projeto, que está em curso desde 1997 e tem como ponto-chave para o seu sucesso a ampla captação de mulheres, a ampliação de recursos humanos para a coleta do exame citopatológico e a organização do fluxo e dos serviços de maior complexidade para o tratamento do câncer do colo do útero ${ }^{4}$. O protocolo do Programa Viva Mulher estabelece que todas as mulheres sexualmente ativas da área de abrangência de uma Unidade Básica de Saúde devem ser recrutadas em todas as oportunidades onde tiverem contato com o serviço para a realização do exame citopatológico a cada três anos após dois resultados negativos, com intervalo anual. Se o resultado citolpatológico for compatível com lesão intraepitelial de baixo grau ou com células escamosas atípicas de significado indeterminado, provavelmente náo neoplásicas, a coleta do exame deve ser repetida em seis meses 5 . Se a citopatologia evidenciar alteraçôes compatíveis com células escamosas atípicas de significado indeterminado, quando náo se pode excluir lesão intraepitelial de alto grau, células glandulares atípicas de significado indeterminado, tanto para as possivelmente não neoplásicas quanto para aquelas em que não se pode excluir lesão de alto grau; células atípicas de origem indefinida, possivelmente não neoplásicas e quando não se pode excluir lesão de alto grau; lesão intraepitelial de alto grau; lesão intraepitelial de alto grau não podendo excluir microinvasão ou carcinoma epidermoide invasor; adenocarcinoma in situ e invasor, a mulher deve ser encaminhada a uma referência secundária, onde será submetida à colposcopia. Quando a colposcopia demonstra lesão, esta é removida por meio da cirurgia de alta frequência $(\mathrm{CAF})$ e a peça cirúrgica enviada para a análise histopatológica. $\mathrm{O}$ resultado encontrado no exame histopatológico fundamenta a escolha da terapêutica ou seguimento adequado para as mulheres portadoras de lesôes neoplásicas no colo do útero ${ }^{4,6}$.

Em alguns casos, a análise histológica das peças removidas pode sofrer vieses de interpretaçáo e o diagnóstico correto pode ser difícil. As neoplasias intraepiteliais cervicais, por exemplo, graduadas de acordo com a altura do epitélio ocupado por células imaturas, poderiam ser erroneamente analisadas devido à presença de artefatos de inclusão na preparação do espécime ${ }^{6}$.

O diagnóstico histopatológico de NIC II parece ser um dos mais difíceis de ser identificado, uma vez que ele é representado pela "presença de células neoplásicas nos dois terços inferiores da espessura do epitélio, poupando o terço superior"7 ${ }^{17}$, podendo esse diagnóstico ser afetado tanto pela acurácia da preparação da peça histológica quanto pela interpretaçáo do patologista ${ }^{8}$. Um estudo de controle de qualidade realizado nos Estados Unidos demonstrou que a maioria das mulheres diagnosticadas com NIC II na biópsia realizada no centro onde foram atendidas recebeu diagnósticos diferentes quando foram avaliadas pelo grupo de controle de qualidade de Patologia ${ }^{6}$.

O controle de qualidade é parte fundamental do processo de garantia de um serviço, para que este beneficie ao máximo os clientes que dele dependem; processo este que também envolve oportunidade, precisão, comunicação efetiva e a consolidação dos objetivos propostos 9 . No controle de qualidade, avaliam-se os processos que levam ao diagnóstico histopatológico.

No estado do Paraná, os resultados da análise histopatológica das biópsias e peças removidas pela CAF sáo analisados por patologistas nos laboratórios credenciados e o laudo do diagnóstico é enviado junto com uma lâmina representativa da lesão para a Unidade de Monitoramento Externo da Qualidade, vinculada à Associação Paranaense de Patologia (UMEQ-APP), responsável por revisar o diagnóstico inicial e realizar o controle de qualidade dos serviços de diagnóstico de lesôes cervicais de todo o estado. A revisão baseia-se na distribuição em grupos das lesôes: lesôes benignas, lesões intraepiteliais de baixo grau, lesôes intraepiteliais de alto grau e lesôes invasoras, sendo considerado discordância quando há modificação de um dos grupamentos para 
outro. Os casos são analisados pelos membros da UMEQ do programa de prevenção e nos casos duvidosos pelo menos dois patologistas avaliam os preparados.

Uma vez que o seguimento e o tratamento das mulheres submetidas à excisão de lesão cervical por CAF é dependente do diagnóstico histopatológico da peça removida, é fundamental que não só a análise realizada no laboratório da rede de serviço seja correta, mas também os mecanismos de controle de qualidade desse serviço sejam precisos e efetivos.

Este trabalho tem por objetivo comparar o diagnóstico inicial de biópsias e peças cirúrgicas removidas por CAF fornecido por um laboratório da rede de serviço com o diagnóstico revisado pela UMEQ-APP, identificando quais foram as discrepâncias mais prevalentes.

\section{MÉTODO}

Foi realizado um estudo do tipo transversal observacional, analisando os laudos do banco de dados da UMEQ-APP referentes ao ano de 2010. Destes casos, recolheram-se dados das mulheres, dos laboratórios iniciais e dos diagnósticos de biópsias e produtos de CAF, tanto do laboratório da rede de serviço quanto do controle de qualidade, verificando-se também a concordância ou discordância entre ambos.

Os diagnósticos na unidade são catalogados em grandes grupos, relacionados com a forma de acompanhamento ou terapêutica complementar indicada em cada caso e abrangeram: processos patológicos benignos (pólipos endocervicais, cervicite crônica inespecífica, metaplasia escamosa e demais alteraçóes de caráter benigno), lesóes intraepiteliais de baixo grau ou LIEBG (lesōes compreendendo efeito citopático pelo vírus HPV e NIC I), lesóes intraepiteliais de alto grau ou LIEAG (NIC II e NIC III), carcinoma epidermoide invasor, adenocarcinoma in situ e invasor, além de outras neoplasias (incluídos neste grupo os casos onde uma única peça conteve mais de uma neoplasia). Após a coleta desses dados, os resultados encontrados foram submetidos à análise estatística descritiva. Para avaliar o grau de concordância entre as duas observaçôes, utilizou-se o índice Kappa.

Este trabalho foi submetido à avaliação pelo Comitê de Ética em Pesquisa da Faculdade Evangélica do Paraná, sendo aprovado com o protocolo número 5644/11.

\section{RESULTADOS}

Foram avaliados 759 laudos vindos de 16 laboratórios de diferentes localidades do estado do Paraná. Em relação às mulheres, as idades variaram entre $14 \mathrm{e} 99$ anos, sendo a média de idade igual a 37,2 anos. De acordo com os diagnósticos fornecidos pelo Controle de Qualidade, o diagnóstico mais encontrado foi o de lesão intraepitelial de alto grau, (48,4\%), seguido pelo de patologias benignas $(34,5 \%)$, pelo de lesão intraepitelial de baixo grau $(13,3 \%)$, pelo de carcinoma epidermoide invasor $(2,4 \%)$, outras neoplasias $(0,8 \%)$, adenocarcinoma in situ $(0,4 \%)$ e adenocarcinoma invasor $(0,3 \%)$

Dos 759 laudos avaliados, 748 tiveram os diagnósticos do laboratório da rede de serviço e do controle de qualidade concordantes, o que confere um percentual de concordância de 98,6\%. Apenas 11 laudos foram discordantes.

A discordância diagnóstica mais encontrada foi na categoria LIEBG em relação ao laboratório da rede de serviço, sendo o diagnóstico redirecionado para patologia benigna (mais frequentemente cervicite crônica inespecífica e metaplasia escamosa) no controle de qualidade, correspondendo a $36,3 \%$ da discordância diagnóstica. Em segundo lugar, a discrepância mais comum foi entre diagnóstico inicial de LIEBG com diagnóstico do controle de qualidade de LIEAG, que correspondeu a $27,3 \%$ das discordâncias. Em terceiro lugar, com um percentual de $18,2 \%$, apareceu o diagnóstico de LIEAG inicial corrigido para patologia benigna pelo controle de qualidade. Respondendo por 9,1\% do percentual de discordância cada, apareceram os diagnósticos iniciais de patologia benigna e posterior de LIEAG e o diagnóstico inicial de LIEAG e posterior de carcinoma epidermoide invasor. As discordâncias diagnósticas mais comuns encontram-se na Tabela 1. A Tabela 2 demonstra a comparação entre todos os diagnósticos iniciais e finais estudados neste trabalho. A Figura 1 exemplifica dois casos de discordâncias encontrados. No primeiro, a lesão recebeu o diagnóstico inicial de NIC I (LIEBG) devido à presença de atipias

Tabela 1. Erros diagnósticos encontrados

\begin{tabular}{|c|c|c|c|}
\hline $\begin{array}{l}\text { Diagnóstico } \\
\text { LRS' }\end{array}$ & CQ" & Absoluto & Percentual \\
\hline LIEBG & $\begin{array}{l}\text { Patologia } \\
\text { benigna }\end{array}$ & 4 & $36,3 \%$ \\
\hline LIEBG & LIEAG & 3 & $27,3 \%$ \\
\hline LIEAG & $\begin{array}{l}\text { Patologia } \\
\text { benigna }\end{array}$ & 2 & $18,2 \%$ \\
\hline $\begin{array}{l}\text { Patologia } \\
\text { benigna }\end{array}$ & LIEAG & 1 & $9,1 \%$ \\
\hline LIEAG & $\begin{array}{l}\text { Carcinoma } \\
\text { epidermoide } \\
\text { invasor }\end{array}$ & 1 & $9,1 \%$ \\
\hline \multicolumn{2}{|c|}{ TOTAL } & 11 & $100 \%$ \\
\hline
\end{tabular}

I: Laboratório da rede de serviço; II: Controle de qualidade 
no epitélio de superfície, identificadas posteriormente como atipias de caráter inflamatório e de reparação pelo controle de qualidade, sendo o diagnóstico revisado para cervicite crônica inespecífica e este representando um caso de supradiagnóstico. No segundo exemplo, a lesão foi diagnosticada pelo controle de qualidade como NIC II (LIEAG), tendo sido diagnosticada pelo laboratório da rede de serviço como NIC I devido à presença de coilócitos no terço superior do epitélio, caracterizando subdiagnóstico.

O grau de concordância para cada diagnóstico foi calculado através do índice Kappa. Para todas as categorias estudadas, encontrou-se um valor de Kappa igual a 0,99, o que é considerado excelente. Os valores de Kappa encontrados estáo descritos na Tabela 3.

Tabela 2. Comparação de diagnósticos entre o LRS' e o CQ"

\begin{tabular}{|c|c|c|c|c|c|c|c|c|c|}
\hline \multicolumn{10}{|c|}{ DIAGNÓSTICO FINAL (CQ) } \\
\hline \multirow{2}{*}{\multicolumn{2}{|c|}{ DIAGNÓSTICO INICIAL (LRS) }} & \multicolumn{8}{|c|}{ Neoplasias malignas } \\
\hline & & $\begin{array}{l}\text { Patologia } \\
\text { benigna }\end{array}$ & LIEBG & LIEAG & $\begin{array}{c}\text { Ca. escamoso } \\
\text { invasor }\end{array}$ & $\begin{array}{l}\text { Adenoca. } \\
\text { in situ }\end{array}$ & $\begin{array}{c}\text { Adenoca. } \\
\text { invasor }\end{array}$ & $\begin{array}{c}\text { Outras } \\
\text { neoplasias }\end{array}$ & TOTAL \\
\hline \multirow{4}{*}{$\begin{array}{l}\text { ․ㅡㅁ } \\
\text { 흘 } \\
\text { ․ㅡㅁ } \\
\text { 응 } \\
\text { 흠 }\end{array}$} & $\begin{array}{l}\text { Cervicite crônica } \\
\text { inespecífica } \\
\% \\
\end{array}$ & $\begin{array}{c}58 \\
(22,1)\end{array}$ & 0 & 0 & 0 & 0 & 0 & 0 & 58 \\
\hline & $\begin{array}{l}\text { Cervicite crônica } \\
\text { inespecífica }+ \\
\text { metaplasia escamosa } \\
\%\end{array}$ & $\begin{array}{c}112 \\
(42,7)\end{array}$ & 0 & $\begin{array}{c}1 \\
(0,3)\end{array}$ & 0 & 0 & 0 & 0 & 113 \\
\hline & $\begin{array}{l}\text { Metaplasia escamosa } \\
\%\end{array}$ & $\begin{array}{c}13 \\
(5,0)\end{array}$ & 0 & 0 & 0 & 0 & 0 & 0 & 13 \\
\hline & $\begin{array}{l}\text { Pólipo endocervical } \\
\%\end{array}$ & $\begin{array}{c}73 \\
(27,9)\end{array}$ & 0 & 0 & 0 & 0 & 0 & 0 & 73 \\
\hline \multirow{4}{*}{ 总 } & $\begin{array}{l}\text { Condiloma acuminado } \\
\%\end{array}$ & 0 & $\begin{array}{c}1 \\
(1,0)\end{array}$ & 0 & 0 & 0 & 0 & 0 & 1 \\
\hline & $\begin{array}{l}\text { Cervicite + indícios de } \\
\text { presença de HPV } \\
\% \\
\end{array}$ & 0 & $\begin{array}{c}8 \\
(7,9)\end{array}$ & $\begin{array}{c}1 \\
(1)\end{array}$ & 0 & 0 & 0 & 0 & 9 \\
\hline & $\begin{array}{l}\text { LIEBG } \\
\%\end{array}$ & 0 & $\begin{array}{c}1 \\
(1,0)\end{array}$ & 0 & 0 & 0 & 0 & 0 & 1 \\
\hline & $\begin{array}{l}\mathrm{NICl} \\
\% \\
\end{array}$ & $\begin{array}{c}4 \\
(1,5) \\
\end{array}$ & $\begin{array}{c}91 \\
(90,1)\end{array}$ & $\begin{array}{c}2 \\
(0,5) \\
\end{array}$ & 0 & 0 & 0 & 0 & 97 \\
\hline \multirow{2}{*}{ 岕 } & $\begin{array}{l}\text { NIC II } \\
\%\end{array}$ & $\begin{array}{c}1 \\
(0,4)\end{array}$ & 0 & $\begin{array}{c}162 \\
(44,1)\end{array}$ & 0 & 0 & 0 & 0 & 163 \\
\hline & $\begin{array}{l}\text { NIC III } \\
\% \\
\end{array}$ & $\begin{array}{c}1 \\
(0,4)\end{array}$ & 0 & $\begin{array}{c}201 \\
(54,8)\end{array}$ & $\begin{array}{c}1 \\
(5,6) \\
\end{array}$ & 0 & 0 & 0 & 203 \\
\hline \multirow{4}{*}{ 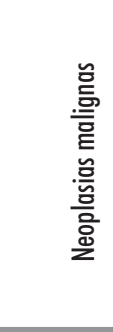 } & $\begin{array}{l}\text { Ca. escamoso inv. } \\
\%\end{array}$ & 0 & 0 & 0 & $\begin{array}{c}17 \\
(94,4)\end{array}$ & 0 & 0 & 0 & 17 \\
\hline & $\begin{array}{l}\text { Adenoca. in situ } \\
\%\end{array}$ & 0 & 0 & 0 & 0 & $\begin{array}{c}3 \\
(100) \\
\end{array}$ & 0 & 0 & 3 \\
\hline & $\begin{array}{l}\text { Adenoca. invasor } \\
\%\end{array}$ & 0 & 0 & 0 & 0 & 0 & $\begin{array}{c}2 \\
(100) \\
\end{array}$ & 0 & 2 \\
\hline & $\begin{array}{l}\text { Outras neoplasias } \\
\%\end{array}$ & 0 & 0 & 0 & 0 & 0 & 0 & $\begin{array}{c}6 \\
(100)\end{array}$ & 6 \\
\hline \multicolumn{2}{|l|}{ TOTAL } & $\begin{array}{c}262 \\
(100)\end{array}$ & $\begin{array}{c}101 \\
(100)\end{array}$ & $\begin{array}{c}367 \\
(100)\end{array}$ & $\begin{array}{c}18 \\
(100)\end{array}$ & $\begin{array}{c}3 \\
(100)\end{array}$ & $\begin{array}{c}2 \\
(100)\end{array}$ & $\begin{array}{c}6 \\
(100)\end{array}$ & 759 \\
\hline
\end{tabular}

I: Laboratório da rede de serviço; II: Controle de qualidade

Tabela 3. Grau de concordância entre diagnósticos

\begin{tabular}{|l|l|c|c|c}
\hline & Patologia benigna & LIEBG & LIEAG & Neoplasias malignas \\
\hline Índice Kappa & 0,99969 & 0,99978 & 0,99966 & 0,99982 \\
\hline
\end{tabular}

Para este trabalho, considerou-se a seguinte relação entre o valor de Kappa e a concordância: 0: pobre; 0 - 0,2 ligeira; 0,21 - 0,4: considerável; 0,41 - 0,6: moderada; 0,61 - 0,8: substancial; $0,81-1$ : excelente 
Também se avaliou a acurácia diagnóstica para cada laboratório, de forma individual, conforme demonstrado na Tabela 4. O percentual de concordância variou entre $100 \%$ e $92,9 \%$, com mediana igual a $100 \%$ e moda também igual a $100 \%$. A média do percentual de concordância foi de $98,6 \%$.

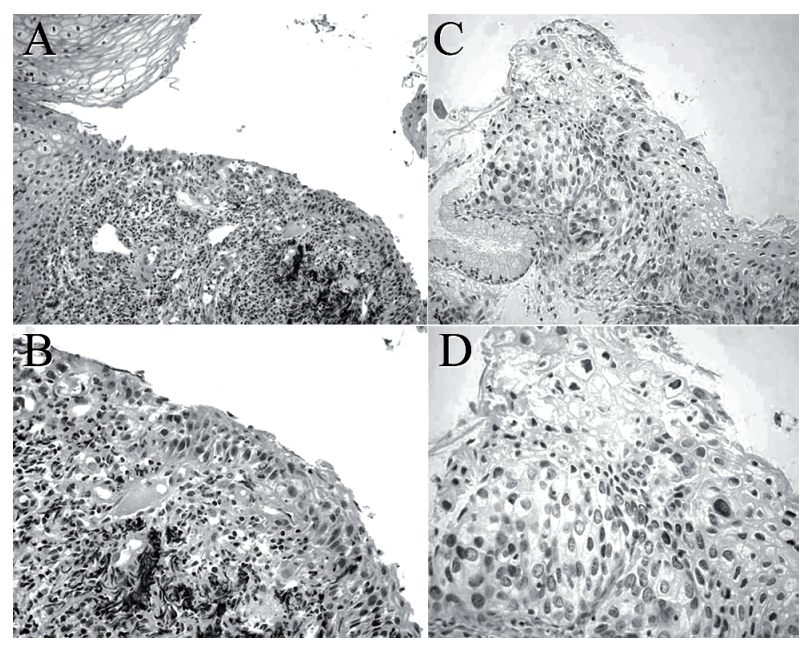

Figura 1. Dois dos casos de discordância diagnóstica encontrados. As figuras A e B representam, em maior e menor aumento, o caso inicialmente diagnosticado como LIEBG e corrigido para cervicite crônica inespecífica, enquanto as figuras $C$ e $D$ representam, também, em maior e menor aumento, a lesão inicialmente caracterizada como LIEBG e corrigida para LIEAG

\section{DISCUSSÃO}

A concordância entre o diagnóstico inicial e o emitido pelo UMEQ-APP de 98,6\% encontrada neste trabalho foi maior do que a encontrada em outros estudos realizados nos Estados Unidos, entre 63,4\%8 e 66,8\% ${ }^{6}$. A taxa de concordância individual de todos os laboratórios também se manteve bem acima desses índices. Tal fato pode ser explicado porque tais estudos usaram uma classificação diferente para os diagnósticos: ao invés do consenso de lesôes de baixo e alto grau, patologias benignas e neoplasias invasoras adotado neste trabalho, considerou-se de forma separada os diagnósticos de cada tipo de neoplasia intraepitelial o que diminui a reprodutibilidade diagnóstica.

Se neste estudo considerássemos cada NIC como uma entidade separada, é possível que a nossa taxa de concordância também fosse menor, no entanto, classificar as lesões em LIEBG ou LIEAG já permite a definição de uma conduta correta para as portadoras de lesóes 5 . Além disso, tais estudos associaram a análise histológica à genotipagem do HPV, o que aumenta o valor preditivo positivo do diagnóstico de presença de infecção pelo HPV.

Há ainda autores que afirmam que talvez a NIC II não seja uma entidade biológica verdadeira, mas sim um diagnóstico equivocado de neoplasia in situ ${ }^{6}$, significando tanto uma lesão pré-cancerígena quanto a infecção por HPV. De acordo com essa teoria, a NIC II seria

Tabela 4. Controle de Qualidade por Laboratório

\begin{tabular}{l|c|c|c|c}
\hline \multicolumn{1}{c|}{ Laboratório } & Laudo concordante & Laudo discordante & Total absoluto & $\begin{array}{c}\text { Percentual de } \\
\text { concordância }\end{array}$ \\
\hline Laboratório 1 & 158 & 2 & 160 & $98,75 \%$ \\
\hline Laboratório 2 & 119 & 4 & 123 & $96,7 \%$ \\
\hline Laboratório 3 & 90 & 0 & 90 & $100 \%$ \\
\hline Laboratório 4 & 89 & 0 & 89 & $100 \%$ \\
\hline Laboratório 5 & 78 & 1 & 79 & $98,7 \%$ \\
\hline Laboratório 6 & 49 & 0 & 49 & $100 \%$ \\
\hline Laboratório 7 & 42 & 0 & 42 & $100 \%$ \\
\hline Laboratório 8 & 29 & 1 & 30 & $96,7 \%$ \\
\hline Laboratório 9 & 20 & 0 & 20 & $100 \%$ \\
\hline Laboratório 10 & 14 & 1 & 15 & $93,3 \%$ \\
\hline Laboratório 11 & 13 & 1 & 14 & $92,9 \%$ \\
\hline Laboratório 12 & 13 & 1 & 14 & $92,9 \%$ \\
\hline Laboratório 13 & 14 & 0 & 14 & $100 \%$ \\
\hline Laboratório 14 & 12 & 0 & 12 & $100 \%$ \\
\hline Laboratório 15 & 4 & 0 & 4 & $100 \%$ \\
\hline Laboratório 16 & 4 & 0 & 4 & $100 \%$ \\
\hline TOTAL & 748 & 11 & 759 & $98,6 \%$ \\
\hline
\end{tabular}


consequência da inacurácia das colposcopias e biópsias guiadas por colposcopia, o que representaria uma visão menos acurada do quadro real da mulher.

$O$ equívoco diagnóstico mais encontrado nesta casuística foi a confusão entre o diagnóstico de LIEBG (NIC I e atipia compatível com infecção por HPV) pelo laboratório da rede de serviço com posterior correção para cervicite crônica inespecífica (acompanhada em alguns casos por metaplasia escamosa), um processo patológico benigno. Tal achado diferiu do encontrado na literatura, onde erros percentualmente mais comuns foram o diagnóstico inicial de normalidade com correção para NIC I ${ }^{6}$ e o diagnóstico de NIC II corrigido posteriormente para NIC III ${ }^{8}$. No entanto, pode-se considerar que as alteraçóes inflamatórias são conhecidamente alteraçôes simuladoras de lesôes de baixo grau à histopatologia e soma-se o fato de que o patologista do laboratório da rede de serviço pode ter sido influenciado por resultado de citopatologia prévia que a mulher possuísse, viés este que os revisores do controle de qualidade não estão expostos.

Já a confusão discriminatória entre NIC II e NIC III não apresentaria tanto prejuízo às mulheres, uma vez que o tratamento e seguimento são os mesmos para ambos os casos 5 . No entanto, a improcedência no diagnóstico entre normalidade e NIC I ou, como foi encontrado no presente estudo, entre LIEAG e carcinoma epidermoide invasor são mais graves, já que representam subdiagnóstico e podem aumentar a morbidade e mortalidade das portadoras das lesóes ${ }^{10}$.

A literatura destaca também que o preparo histológico pode influenciar a interpretação da lâmina ${ }^{6}$, uma vez que a espessura e orientação do corte poderiam influenciar na avaliação do patologista. Num dos casos em que o diagnóstico foi discordante, havia uma observação do examinador dizendo que "sua análise havia sido prejudicada pela qualidade precária da lâmina que havia sido enviada". Assim, pode-se inserir no viés de preparo, além do corte do material ao micrótomo, a fixação e preservação adequada das peças removidas, num contexto que extrapola o laboratório de histopatologia e envolve também os profissionais responsáveis pela excisão das lesôes. A coleta do material já foi indicada como um fator determinante nas discrepâncias diagnósticas em citopatologia ${ }^{10}$ e é possível que também o seja na histopatologia. Vários trabalhos ${ }^{10-12}$ descrevem a variação diagnóstica entre diferentes observadores na citopatologia, podendo-se inferir que a interpretação e experiência de cada patologista também sejam um fator determinante para as discordâncias diagnósticas na histopatologia, relacionadas à obtenção, preparo do material e proficiência do patologista encarregado da leitura do exame. Torna-se fundamental, portanto, educação continuada, treinamento e sistematização dos diagnósticos para os patologistas que analisarão as biópsias, bem como o treinamento e orientação para os médicos encarregados da obtenção e envio do material.

Ressaltamos ainda a importância do controle de qualidade, tal qual é realizado no programa de prevenção do câncer do colo do útero e mama no Estado do Paraná, pois trata-se de uma peça-chave para a melhoria da qualidade diagnóstica e bom atendimento a população, uma vez que permite a identificação das discordâncias diagnósticas e suas causas bem como definição de estratégias para o direcionamento de educaçáo continuada e aprimoramento diagnóstico.

\section{CONCLUSÃO}

Em relação ao controle de qualidade da histopatologia dos produtos de biópsias e cirurgias de alta frequência na presente amostra, pode-se concluir que houve concordância de 98,6\% entre os diagnósticos originais dos laboratórios credenciados e a UMEQ-APP, variando entre $100 \%$ e $92,9 \%$ nos laboratórios avaliados.

Os erros mais encontrados foram no grupo de LIEBG com direcionamento para patologia benigna (supradiagnóstico) e LIEBG sendo direcionado LIEAG (subdiagnóstico).

As taxas de concordância encontradas são excelentes, com índice Kappa igual 0,99, e comprovam a eficácia dos exames realizados.

\section{AGRADECIMENTOS}

À APP pela colaboração e disponibilização do seu banco de dados para a realização deste trabalho.

\section{CONTRIBUIÇÕES}

Larissa Uhlmann Wendling e Luiz Martins Collaço contribuíram na concepção e planejamento do projeto de pesquisa; na obtenção e/ou análise e interpretação dos dados; na redação e revisão crítica. Ana Paula Martins Sebastião, Avelino Hass, Joel Totsugui, Lucia de Noronha e Samuel Regis Araújo contribuíram na obtenção e/ou análise e interpretação dos dados.

Declaraçáo de Conflito de Interesses: Nada a Declarar.

\section{REFERÊNCIAS}

1. Instituto Nacional de Câncer José Alencar Gomes da Silva. Estimativa 2012: incidência de câncer no Brasil [Internet]. Rio de Janeiro: INCA; 2011 [citado 2012 maio 16]. 118 p. Disponível em: http://www.inca.gov. br/estimativa/2012/estimativa20122111.pdf 
2. Motta EV, Fonseca AM, Bagnoli VR, Ramos LO, Pinotti JA. Colpocitologia em ambulatório de ginecologia preventiva. Rev Assoc Med Bras. 2001; 47(4):302-10.

3. Pinho AA, Mattos MCFI. Validade da citologia cervicovaginal na detecção de lesões pré-neoplásicas e neoplásicas de colo de útero. J bras patol med lab. 2002;38(3):225-31.

4. Secretaria Municipal de Saúde (Curitiba). Programa Viva Mulher em Curitiba - Controle do câncer de mama e colo de útero. Curitiba; 2002. 27 p.

5. Lapin GA, Derchain SFM, Tambascia J. Comparação entre a colpocitologia oncológica de encaminhamento e a da gravidade das lesões cervicais intra-epiteliais. Rev Saúde Públ. 2000;34(2):120-5.

6. Castle PE, Stoler MH, Solomon D, Schiffman M. The relationship of community biopsy-diagnosed cervical intraepithelial neoplasia grade 2 to the quality control pathology-reviewed diagnoses: an ALTS report. Am J Clin Pathol. 2007;127(5):805-15.

7. Crum CP. Aparelho genital feminino. In: Kumar V, Abbas AK, Fausto N, [editores]. Robbins e Cotran patologia: bases patológicas das doenças. 7a ed. Rio de Janeiro: Elsevier; 2005. p. 1105-67.

8. Galgano MT, Castle PE, Stoler MH, Solomon D, Schiffman M. Can HPV-16 genotyping provide a benchmark for cervical biopsy specimen interpretation? Am J Clin Pathol. 2008;130(1):65-70.

9. Ong BB, Milne N. Quality assurance in forensic pathology. Malays J Pathol. 2009;31(1):17-22.

10. Di Loreto C, Maeda MYS, Utagawa ML, Longatto Filho A, Alves VAF. Garantia de qualidade em citopatologia: aspectos da correlação cito-histopatológica. Rev Assoc Med Bras. 1997;43(3):195-8.

11. O'Sullivan JP, Ismail SM, Barnes WS, Deery AR, Gradwell E, Harvey JA, et al. Interobserver variation in the diagnosis and grading of dyskaryosis in cervical smears: specialist cytopathologists compared with nonspecialists. J Clin Pathol. 1994;47(6):515-8.

12. Munhoz LMBS, Colturato PL, Borba TAG, Gonçalves S, Merlin JC, Haas P. Comparativo citológico, colposcópico e histológico de biópsias do colo uterino no ambulatório Amaral Carvalho/Itararé-SP. Rev bras anal clin. 2009;41(3):167-71. 


\section{Abstract}

Introduction: Proper monitoring and treatment of neoplastic and pre-neoplastic cervical lesions depend of the correct histopathological diagnosis. The establishment of mechanisms for quality control for this diagnostic procedure is essential for guaranteeing precision to the exams. Objective: To compare the first diagnosis of biopsies and surgical specimens removed by loop electrosurgical excisional procedure, given by a laboratory of the basic network, with the reviewed diagnosis given by the Quality Control Center from the Associação Paranaense de Patologia, identifying the most common diagnostic mistakes. Method: An observational cross-sectional study was carried out with a databank review from the Quality Control Center, regarding the year 2010, in order to evaluate the general agreement percentage for each laboratory and the most common disagreements in diagnosis of biopsies and surgical specimens from loop electrosurgical excisional procedure of the cervix. Concordance rate was measured with Kappa Index. Results: The agreement rate between first and quality control diagnoses was $98.6 \%$, which varied among laboratories between $92.9 \%$ and $100 \%$. The most common diagnosis discrepancies found were between low-grade squamous intraepithelial lesion and cervicitis (36.3\%), and low-grade squamous intraepithelial lesion and high-grade squamous intraepithelial lesion (27.3\%). For all the diagnostic categories studied, the Kappa Index was 0.99. Conclusion: The most common misdiagnoses were between low-grade squamous intraepithelial lesion and cervicitis (overdiagnosis) and between low-grade squamous intraepithelial lesion and high-grade squamous intraepithelial lesion (underdiagnosis). The high agreement rate found was considered excellent.

Key words: Quality Control; Uterine Cervical Neoplasms/diagnosis; Uterine Cervical Neoplasms/pathology; Precancerous Conditions/diagnosis; Conization; Cervical Intraepithelial Neoplasia/pathology

\section{Resumen}

Introducción: El seguimiento y tratamiento adecuado de las lesiones neoplásicas y preneoplásicas del cuello del útero dependen del diagnóstico histopatológico correcto. La introducción de mecanismos de control de calidad para este procedimiento diagnóstico es fundamental para garantizar la precisión a las pruebas. Objetivo: Equiparar el diagnóstico inicial de biopsias y piezas quirúrgicas removidas por Cirugía de Alta Frecuencia, suministrado por un laboratorio de la red de servicio, con el diagnóstico repasado por la unidad de supervisión de calidad de la Associação Paranaense de Patologia, identificando cuales las diferencias más relevantes. Método: Estudio transversal observacional con revisión de la base de datos de la unidad de Control de Calidad referente al año de 2010 para evaluar el percentual de concordancia general, para cada laboratorio y las diferencias diagnósticas más comunes en productos de biopsias y cirugías de alta frecuencia del cuello del útero. La concordancia fue evaluada a través del índice Kappa. Resultados: La compatibilidad entre el diagnóstico inicial y el control de calidad fue del 98,6\%, oscilando por laboratorio entre $92,9 \%$ y $100 \%$. Las discrepancias diagnósticas se encuentran entre la lesión intraepitelial de bajo grado y cervicitis $(36,3 \%)$ y lesión intraepitelial de bajo grado y lesión intraepitelial de alto grado (27,3\%). Para todas las categorías diagnósticas estudiadas se encontró el índice de Kappa de 0,99. Conclusión: Las discrepancias diagnósticas más comunes han sido entre lesión intraepitelial de bajo grado y diagnóstico de lesiones benignas (sobrediagnósticos) y entre la lesión intraepitelial de bajo grado y lesión intraepitelial de alto grado (subdiagnóstico). Las tasas de concordancia encontradas son consideradas excelentes.

Palabras clave: Control de Calidad; Neoplasias del Cuello Uterino/diagnóstico; Neoplasias del Cuello Uterino/ patología; Lesiones Precancerosas/diagnóstico; Conización; Neoplasia Intraepitelial del Cuello Uterino/patología 